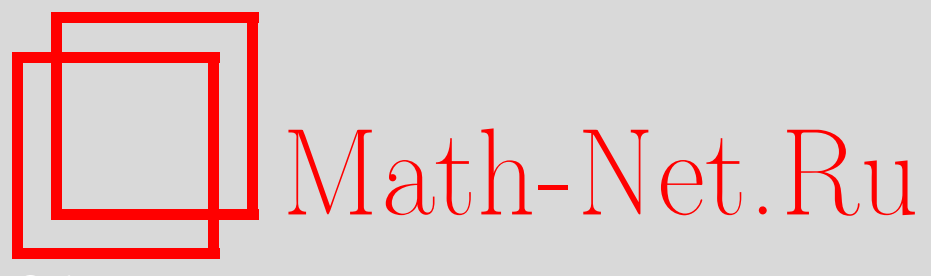

Ф. Б. Пакович, Об аналогах теорем Ритта для рациональных функций с двумя полюсами, УМН, 2008, том 63, выпуск 2, 181-182

DOI: https://doi.org/10.4213/rm9065

Использование Общероссийского математического портала Math-Net.Ru подразумевает, что вы прочитали и согласны с пользовательским соглашением http://www . mathnet.ru/rus/agreement

Параметры загрузки:

IP : 54.198 .64 .247

26 апреля 2023 г., 12:06:46

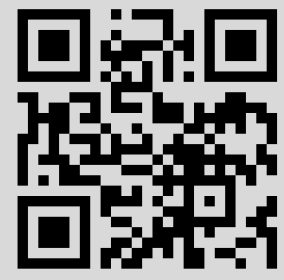




\section{Об аналогах теорем Ритта для рациональных функций с двумя полюсами}

\section{Ф. Б. Пакович}

Пусть $F$ - рациональная функция с комплексными коэффициентами. Такая функция $F$ называется неразложимой, если равенство $F=F_{1} \circ F_{2}$, где $F_{1}, F_{2}$ - рациональные функции, а $F_{1} \circ F_{2}$ обозначает суперпозицию $F_{1}\left(F_{2}(z)\right)$, влечет, что, по крайней мере, одна из функций $F_{1}, F_{2}$ является преобразованием Мёбиуса. Любая рациональная функция $F$ может быть разложена в композицию неразложимых рациональных функций, вообще говоря, не единственным образом. Такие разложения называются максимальными. Два разложения $F=F_{1} \circ \cdots \circ F_{r}$ и $F=G_{1} \circ \cdots \circ G_{r}$, максимальные или нет, называются эквивалентными, если существуют преобразования Мёбиуса $\mu_{i}$, $1 \leqslant i \leqslant r-1$, такие, что

$$
F_{1}=G_{1} \circ \mu_{1}, \quad F_{i}=\mu_{i-1}^{-1} \circ G_{i} \circ \mu_{i}, \quad 1<i<r, \quad \text { и } \quad F_{r}=\mu_{r-1}^{-1} \circ G_{r} .
$$

Теория разложений полиномов была построена Дж. Ф. Риттом в его классической статье [1]. Приводимая ниже теорема расширяет теорию Ритта на случай рациональных функций, имеющих не более двух полюсов.

TeOpema. Пусть

$$
L=A \circ C=B \circ D
$$

- разложение рачиональной функиии $L$, имеющей не более двух полюсов, в композицию рациональных функиий $A, B, C, D$. Тогда или разложение $A \circ C$ эквивалентно разложению $B \circ D$, или существуют рациональные функции $U, W, \widetilde{A}, \widetilde{B}, \widetilde{C}, \widetilde{D}$ такие, что

$$
A=U \circ \widetilde{A}, \quad B=U \circ \widetilde{B}, \quad C=\widetilde{C} \circ W, \quad D=\widetilde{D} \circ W, \quad \widetilde{A} \circ \widetilde{C}=\widetilde{B} \circ \widetilde{D}
$$

и, с точностью до замены $A$ на $B$ и $C$ на $D$, выполняется одно из следующих условий:

1) $\widetilde{A} \circ \widetilde{B} \sim z^{n} \circ z^{r} L\left(z^{n}\right), \widetilde{C} \circ \widetilde{D} \sim z^{r} L^{n}(z) \circ z^{n}$, где $L(z)$ - полином Лорана, $r \geqslant 0, n \geqslant 1$ и НОД $(n, r)=1$;

2) $\widetilde{A} \circ \widetilde{C} \sim z^{2} \circ \frac{z^{2}-1}{z^{2}+1} S\left(\frac{2 z}{z^{2}+1}\right), \widetilde{B} \circ \widetilde{D} \sim\left(1-z^{2}\right) S^{2}(z) \circ \frac{2 z}{z^{2}+1}$, где $S(z)$ - полином;

3) $\widetilde{A} \circ \widetilde{C} \sim T_{n} \circ T_{m}, \widetilde{B} \circ \widetilde{D} \sim T_{m} \circ T_{n}$, где $T_{n}(z), T_{m}(z)$ - полиномь Чебышёва, $m, n \geqslant 1$ и НОД $(n, m)=1$;

4) $\widetilde{A} \circ \widetilde{C} \sim T_{n} \circ \frac{1}{2}\left(z^{m}+\frac{1}{z^{m}}\right), \widetilde{B} \circ \widetilde{D} \sim \frac{1}{2}\left(z^{m}+\frac{1}{z^{m}}\right) \circ z^{n}$, где $m, n \geqslant 1$ и НОД $(n, m)=1$;

5) $\widetilde{A} \circ \widetilde{C} \sim-T_{n l} \circ \frac{1}{2}\left(\varepsilon z^{m}+\frac{\bar{\varepsilon}}{z^{m}}\right), \widetilde{B} \circ \widetilde{D} \sim T_{m l} \circ \frac{1}{2}\left(z^{n}+\frac{1}{z^{n}}\right)$,

где $T_{n l}(z), T_{m l}(z)$ - полиномы Чебышёва, $m, n \geqslant 1, l>1, \varepsilon^{n l}=-1 u$ НОД $(n, m)=1$;

6) $\widetilde{A} \circ \widetilde{C} \sim\left(z^{2}-1\right)^{3} \circ \frac{3\left(3 z^{4}+4 z^{3}-6 z^{2}+4 z-1\right)}{\left(3 z^{2}-1\right)^{2}}$,

$$
\widetilde{B} \circ \widetilde{D} \sim\left(3 z^{4}-4 z^{3}\right) \circ \frac{4\left(9 z^{6}-9 z^{4}+18 z^{3}-15 z^{2}+6 z-1\right)}{\left(3 z^{2}-1\right)^{3}} .
$$

Более того, если $\mathscr{D}, \mathscr{E}$ - два максимальных разложения функции $L$, то существует цепочка максимальных разложений $\mathscr{F}_{i}, 1 \leqslant i \leqslant s$, функиии $L$ такая, что $\mathscr{F}_{1}=\mathscr{D}$, $\mathscr{F}_{s} \sim \mathscr{E}$ и $\mathscr{F}_{i+1}$ получается из $\mathscr{F}_{i}$ заменой двух последовательных функиий, входящих в $\mathscr{F}_{i}$, на две другие функиии с той же композищией. 
Полное доказательство теоремы дано в [2]. Отметим, что вторая часть теоремы выводится из первой, а первая часть сводится к анализу уравнений

$$
A\left(L_{1}\right)=B\left(L_{2}\right), \quad A\left(L_{1}\right)=B\left(z^{d}\right),
$$

где $A, B$ - полиномы, а $L_{1}, L_{2}$ - полиномы Лорана. При этом наш анализ первого из этих уравнений дает новое доказательство классификации алгебраических кривых вида $A(x)-B(y)=0$, имеющих фактор рода нуль с двумя точками на бесконечности, полученной в [3], [4]. Отметим также, что наше изложение является полностью самозамкнутым и содержит несколько новых идей, позволяющих упростить подход к проблеме. В частности, мы рассматриваем уравнение (1) в более общем контексте функционального уравнения $f \circ p=g \circ q$, где $f: C_{1} \rightarrow \mathbb{C P}^{1}, g: C_{2} \rightarrow \mathbb{C P}^{1}, p: C \rightarrow C_{1}$, $q: C \rightarrow C_{2}$ - голоморфные функции на компактных римановых поверхностях.

\section{Список литературы}

[1] J. F. Ritt, Trans. Amer. Math. Soc., 23:1 (1922), 51-66. [2] F. Pakovich, arXiv: 0710.3860. [3] Yu. F. Bilu, R. F. Tichy, Acta Arith., 95:3 (2000), 261-288. [4] M. Fried, J. Reine Angew. Math., 264 (1973), 40-55.

Ф. Б. Пакович (F. В. Pakovich)

Ben-Gurion University of the Negev, Israel

E-mail: pakovich@math.bgu.ac.il
Представлено С. К. Ландо Принято редколлегией 07.12 .2007 